\title{
Estrategias Pedagógicas Aplicadas a la Educación con Mediación Virtual para la Generación del Conocimiento Global
}

\author{
Olga Martínez ${ }^{1}$, Ernesto J. Steffens ${ }^{2}$, Decired C. Ojeda ${ }^{3}$ y Hugo G. Hernández ${ }^{3}$ \\ 1 Universidad de la Costa, Dpto. de Humanidades, Calle 58 \# 55-24. Barranquilla-Colombia. \\ (e-mail: omartinez@cuc.edu.co) \\ ${ }^{2}$ Corporación Universitaria Latinoamericana, Facultad de Ciencias Económicas, Calle 58 \# 55-24A \\ Barranquilla-Colombia. (e-mail: steffensse@ul.edu.co) \\ ${ }^{3}$ Universidad del Atlántico, Facultad Ciencias de la Educación, Carrera 30 Número 8- 49. Puerto Colombia- \\ Colombia. (e-mail: desiredojeda22@gmail.com; hugohernandezp@mail.uniatlantico.edu.co)
}

Recibido Dic. 29, 2017; Aceptado Mar. 15, 2018; Versión final Abr. 23, 2018, Publicado Oct. 2018

\section{Resumen}

Se determinan los dominios conceptuales y competencias básicas en estrategias pedagógicas aplicadas a la educación superior con mediación virtual. La investigación desarrollada fue de carácter cuanti-cualitativo de tipo descriptivo aplicada a 55 docentes de cuatro programas académicos en cuatro universidades de la ciudad de Barranquilla en Colombia. Los datos fueron analizados mediante el software estadístico SPSS, y los resultados posibilitaron la generación de alternativas de mejoramiento en la modalidad educativa virtual. Se concluyó que el $52.7 \%$ de los docentes cuentan con un alto nivel de dominio conceptual de las estrategias pedagógicas. Además, que al integrar tres componentes (social, cultural y tecnológica) dentro en las estrategias pedagógicas, estas potencian los procesos de educación superior con mediación virtual.

\section{Pedagogical Strategies Applied to Education with Virtual Media for the Generation of Global Knowledge}

\begin{abstract}
The conceptual domains and basic competences in pedagogical strategies applied to higher education with virtual mediation are determined. The research done was of the qualitative-quantitative type and was applied to a sample of 55 teachers of four universities of the city of Barranquilla in Colombia. The data were analyzed using the statistical software SPSS. The results allowed the generation of alternatives of improvement in the virtual educational modality. It was concluded that $52.7 \%$ of teachers have a high level of conceptual mastery of pedagogical strategies. In addition to integrating three components (social, cultural and technological) within the pedagogical strategies, these enhance the processes of higher education with virtual mediation.
\end{abstract}




\section{INTRODUCCIÓN}

La conjugación de varios elementos durante las últimas dos décadas, como son la vertiginosa evolución de los dispositivos electrónicos y equipos de cómputo, sumados a la expansión del internet y el desarrollo de las tecnologías de la información y las telecomunicaciones (TIC), han propiciado una larga lista de cambios e innovaciones en muchos terrenos de la vida de las personas. De hecho, la mayoría de las cosas han cambiado sustancialmente desde la popularización de estas herramientas tecnológicas; actividades como leer un libro, llevar la contabilidad de una empresa, comprar un objeto que se desea o simplemente consultar una duda o inquietud; se han vuelto cuestiones que se resuelven en poco tiempo, siempre que se tenga a la mano un computador con acceso a internet, mientras que hace quince o veinte años, atrás, estas tareas representaban invertir esfuerzo, tiempo y dinero (Flores, 2014). La educación, no ha sido ajena a estos cambios, la implementación de actividades tecnológicas; permite que los estudiantes conozcan aprendan, se adiestren en la utilización de este tipo de instrumentos, posibilitando el logro de dos objetivos: la asimilación de conocimientos necesarios para su formación y la actualización en la utilización de las innovaciones en las TIC, que se están renovando constantemente (Salguero y Peña, 2015). Esta modalidad educativa, ha ampliado enormemente las posibilidades para quienes no podían estudiar debido a obstáculos como la distancia, los horarios, las ocupaciones laborales o domésticas, pero ya estos perjuicios quedaron atrás; tanto así que la evolución de esta variante formativa, ha generado cambios que han sido necesarios para el desarrollo de esta innovadora modalidad (Sierra, 2013). Como referencia a lo anterior, las escuelas virtuales se están convirtiendo en un fenómeno cuyo crecimiento se ha incrementado en los últimos años en algunos estados de los EEUU por su flexibilidad y características únicas, que proporcionan mecanismos que se ajustan al estilo de vida actual de los estudiantes, transformándose de esta manera, en una tendencia que, de continuar creciendo, representará una alternativa sólida y viable al modelo educativo tradicional (Toppin y Toppin, 2016). Evidencia de esta corriente, ahora en el contexto universitario, se ve refleja en el incremento registrado desde el año 2002, donde apenas el $48,8 \%$ de los líderes académicos señalaban a la educación virtual como crítica y parte fundamental de su estrategia a largo plazo, pasando a un 70,8\% en el 2015 (Allen y Seaman, 2015).

A nivel de Latinoamérica, la preferencia por la educación virtual no es muy diferente, en virtud de ello, el Sistema Educativo Brasileño ha investigado sobre la dinámica y posicionamiento de los Entornos Virtuales de aprendizaje (EVA) como instrumento para el fortalecimiento de los procesos de enseñanza y aprendizaje, en este sentido, la educación a distancia y abierta ofrece la posibilidad para llevar la educación a un mayor número de personas, en sintonía con las políticas públicas del Estado en materia educativa (Gómez, 2014). Dado el importante número de personas interesadas en esta modalidad de aprendizaje, se está haciendo un esfuerzo por que los espacios donde se desarrollan las actividades educativas, cuenten con los requisitos y estándares internacionales de calidad que garanticen su usabilidad técnica (De Pinho et al., 2015).Por lo tanto, esta modalidad de educación presenta retos que sugieren la aplicación de estrategias acordes al contexto, en este caso, es necesario desarrollar una nueva concepción del liderazgo y rol del docente o eleadership que permita crear una comunicación efectiva y un intercambio de información con mayor fluidez, todo ello, con el apoyo de las TIC (García, 2015)

Adicionalmente, no solo las habilidades y competencias del docente deben ser objeto de cambio, sino también sus prácticas. Los modelos para el aprendizaje tienen el compromiso de evolucionar, integrando los elementos característicos del entorno virtual para lograr una inmersión pedagógica que permita evaluar y examinar las etapas del proceso de aprendizaje de acuerdo a los entornos en los que se esté desarrollando (Flower, 2015) Dadas las consideraciones anteriores, se propone hacer una investigación de tipo cualitativo y cuantitativo, que tiene por finalidad, determinar los dominios conceptuales y las competencias básicas en las estrategias pedagógicas aplicadas a la educación superior con mediación virtual, ya que son varias las inserciones y renovaciones que esta realidad ha generado al panorama de educación que acoge esta mediación. En este escenario han surgido algunas modalidades educativas, entre ellas, los cursos masivos virtuales y abiertos a cualquier tipo de usuario denominados Massive Open Online Course (MOOC) por sus siglas en inglés (AlAtabi y De Bóer, 2014), que representan una tendencia con gran potencialidad y con alto impacto en el ámbito educativo actual, tanto que universidades con mejor reputación en Norteamérica tales, como MIT, Harvard, Stanford, entre otras; comenzaron a mediados de 2010, a ofrecer actividades formativas masivas en plataformas virtuales, es así que la masificación de la educación merece especial atención en cuanto a su impacto y valoración (Vázquez, 2014).

Al finalizar la investigación, los resultados obtenidos llevan a pensar que la mayoría de los profesores involucrados en el proceso, están preparados para enfrentar el reto de la modalidad educativa virtual, con una bases bien fundamentadas, pues se logró establecer que un 52,7\% de los docentes que participaron en el proceso, tienen un alto nivel de dominio que tienen sobre los conceptos relacionados con estrategias aplicadas a la pedagogía en la educación virtual, otro $45,5 \%$ de los educadores, registraron un moderado nivel en cuanto a este criterio y sólo en un $1,8 \%$ de los pedagogos, se encontró un bajo nivel de conocimiento, asociado al tema en cuestión. 
La pedagogía, de forma muy general, se puede definir como el conjunto de teorías que orientan a los involucrados en el proceso de enseñanza y aprendizaje, sobre cómo educar tomando en cuenta a los receptores de este proceso (Costa et al., 2015). Desde otra postura, la pedagogía ofrece practicas liberadoras que genera relaciones fuera del ámbito educativo en relaciones bidireccionales y dicotómicas, maestro alumno, padres e hijos, entre otras (Bottero, 2013). Por lo tanto, la pedagogía debe entenderse como un proceso que trasciende la generación de conocimiento, formando sujetos integrales que reconozcan su entorno y su propia individualidad (Recino et al., 2017).

\section{Pedagogía en un contexto virtual}

En un contexto de educación virtual, en el cual no se limita únicamente a las aplicaciones tecnológicas (Bolívar y Dávila, 2016), la pedagogía juega un papel importante en la construcción del aprendizaje significativo (Arroyo et al., 2014). En esta construcción existen elementos del sistema educativo que deben tomarse en cuenta, tales como, el entorno social, cultural y tecnológico, la interacción y actuación de los involucrados en lo virtual con las subjetividades que implica, entre otros aspectos de igual importancia (Rozo, 2011). En función del planteamiento anterior, se puede decir que lo virtual, constituye un ambiente para el desarrollo de competencias bajo una múltiple clasificación de modalidades educativas virtuales donde el centro es el aprendizaje y no la modalidad en sí misma (Díaz y Omara, 2014) por lo tanto, el entorno virtual surge como un nuevo paradigma que está influenciando y promoviendo cambios importantes en los modelos educativos, otorgando flexibilidad y transversalidad al proceso de enseñanza aprendizaje (Edel-Navarro, 2010).

Parte de esos cambios de los modelos educativos, se refieren a que en entornos virtuales de aprendizaje sigue predominando el modelo de transmisión de información (Rubia, 2014) en donde el docente sigue aplicando las prácticas de la educación presencial a través de la transmisión de datos e información con una escasa interacción entre los participantes y poca construcción de conocimiento mediante la colaboración (Fainholc et al., 2015). De este planteamiento surge la llamada pedagogía de la transmisión (Aparici y Silva, 2012). Este modelo pedagógico ha recibido numerosas críticas desde la segunda mitad del siglo XX, dada sus características unidireccionales en donde el modelo comunicacional se basa en emisores y receptores y la retroalimentación se hace como afianzamiento de la información que genera el emisor, por lo que la interacción siempre se da de forma limitada (Aparici y Silva, 2012). La causa de la adopción de este modelo pedagógico, radica en que fue diseñado en el contexto de la revolución industrial (Gómez et al., 2016), en donde la prioridad era cumplir instrucciones para la realización de una tarea o actividad de un proceso en dividido, el cual no estaba conectado con el sistema completo, desde su perspectiva. A raíz de esto, se comienza a plantear estrategias pedagógicas más centradas en los alumnos y la colaboración entre estos (Dusell, 2014). Dusell (2014) sugiere una orientación sobre los niveles o clasificaciones de aprendizaje, propio de modelos pedagógicos más contemporáneos. En la tabla 1 se reflejan las características más relevantes de cada modelo y que se recopilan a manera de ilustración en dos categorías que se consideran de mayor alcance.

Tabla 1: Clasificación de los aprendizajes

\begin{tabular}{|l|l|}
\hline Aprendizaje de masas & Aprendizaje interactivo \\
\hline Con énfasis en el profesor & Con énfasis en el estudiante \\
\hline Directriz: aprender sobre un tema & Enfoque: aprender a ser \\
\hline Aprendizaje autónomo & Aprendizaje colaborativo y de integración \\
\hline
\end{tabular}

La influencia e impacto de las TICS a todo este proceso fue decantando en la transformación de nuevas herramientas pedagógicas (Olea et al., 2016), lo que produjo un nuevo desarrollo económico basado en el conocimiento. Bajo tal esquema, la gran mayoría de las personas están inmersas en la llamada sociedad del conocimiento y no así en la sociedad del aprendizaje (Unzaga et al., 2012). En la figura 1 se ilustra dicha transformación.

Junto con las nuevas herramientas pedagógicas, surgieron también unas nuevas formas de técnicas, métodos y estrategias entre los docentes y la parte organizacional de las instituciones educativas, como el mejoramiento de las comunicaciones a nivel interno, el proceso de adaptación de los contenidos académicos de cada una de las asignaturas o competencias para introducirlas en el entorno virtual, procurando que la información conservara las propiedades que le permiten a los estudiantes el poder asimilar, entender y procesar la información recibida, el compromiso de los docentes por aprender a manejar estas tecnologías o a mejorar los conocimientos que tenía con anterioridad, pues el manejo eficiente de estos equipos, le permitirá tener una interacción más fluida con los estudiantes (Fainholc et al., 2013). 


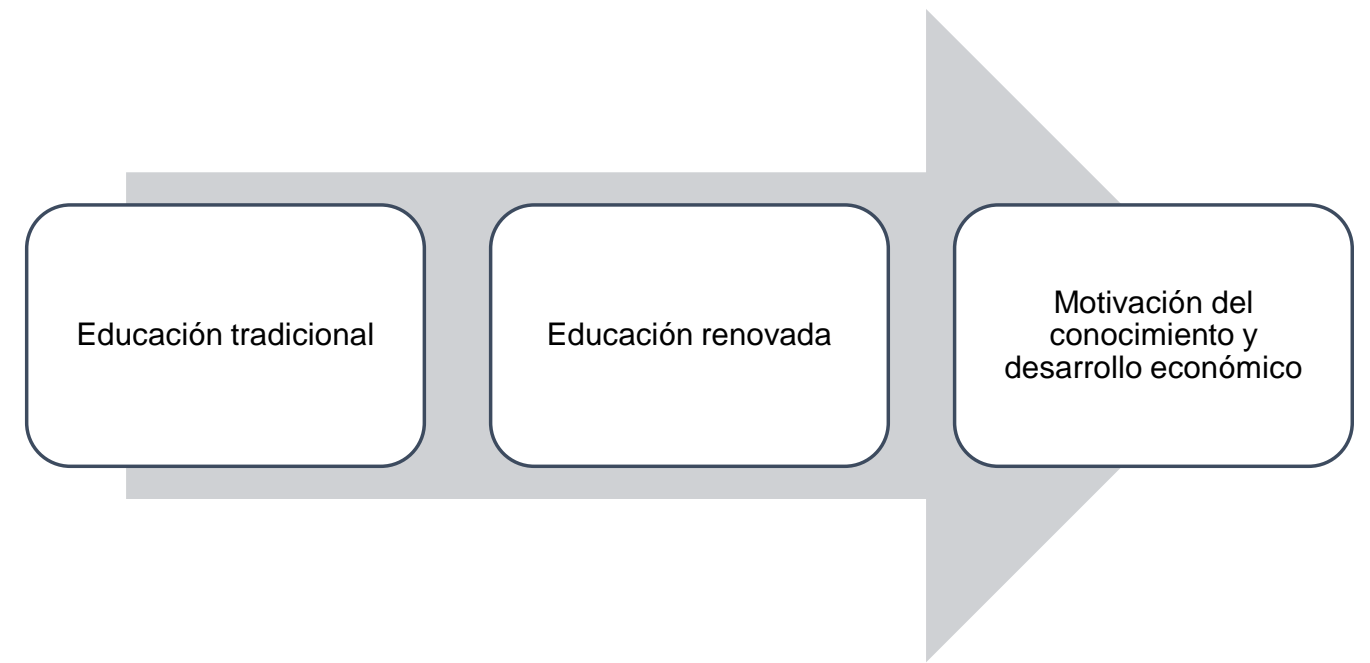

Fig. 1: Proceso de transformación del entorno educativo.

Cabe destacar que este tipo de aprendizajes, presentan una serie de beneficios, como el manejo del tiempo de acuerdo a las necesidades del estudiante, su papel dentro del proceso de enseñanza y aprendizaje, es más dinámico y se evita el aumento de costos que generan los traslados hasta las locaciones de las instituciones educativas, además; con esta modalidad, se pueden ampliar el abanico de alternativas para estudiar, es decir, el espacio virtual, va a posibilitar ofrecer más variedad a la hora de escoger (Gil y Roca, 2011).

\section{El Conectivismo}

Una de las teorías que se han acogido de forma reciente en el análisis de estas tendencias es la denominada teoría de la conectividad, la cual impulsa un enfoque basado en diversas teorías (Martínez y Martínez, 2017) como la teoría del caos, que trata del rompimiento en la armonía de ciertos sistemas matemáticos, con características complejas y dinámica, debido a minúsculas variaciones en los respectivos procedimientos, que obstaculizan la obtención de los resultados requeridos (Sobrino, 2014); la teoría de las redes, que hacen referencia tanto a las redes de aprendizaje, tanto como a los nodos que se pugnan por los enlaces, vitales para la conexión y a las redes de computadores, que son la herramienta clave, dentro del aprendizaje virtual (Henao, 2012); la teoría de la complejidad y de la auto organización, que es la configuración natural de sistemas, modelos y comportamientos, perfectamente estructurados en estados básicos aleatorios (Cardama y Sebastián, 2017), dicha combinación busca darle respuesta a las exigencias tecnológicas de la sociedad actual y explicar cómo los procesos del internet y las redes sociales, han configurado un nuevo discurso social para el mundo y donde las habilidades relacionadas o asociadas han cobrado mayor relevancia (Cárdenas et al., 2013).

El Conectivismo en esencia es una teoría del aprendizaje que dio a conocer Stephen Downes o teoría del aprendizaje para la era digital que explica el aprendizaje complejo en un mundo social digital en rápida evolución (Bartolomé y Steffens, 2015). Esta teoría sugiere que los educadores deben tener en cuenta las conexiones de las redes de nudos y para definir el aprendizaje (Gutiérrez et al., 2015). El Conectivismo se cita entonces como la integración de los principios explorados por el caos en la red y la complejidad y las teorías de la auto-organización. En la figura 2 se observan las alternativas que desde el internet se toman en consideración para la educación en el entorno virtual.

El aprendizaje es un proceso que ocurre dentro de entornos virtuales de elementos básicos que no están totalmente bajo el control del individuo (Marín et al., 2017). El aprendizaje desde esta perspectiva puede estar fuera del individuo, en una base de datos y otro tipo de organización, que conecten información especializada y conexiones que actualizan el conocimiento (Martín et al., 2017). A partir del Conectivismo, el aprendizaje y el conocimiento se estiman articulados en la diversidad de opiniones (Ichimura y Suzuki, 2017). De igual forma, constituye un proceso que conecta nudos especializados, pues los dispositivos pueden, no ser humanos, el proceso apunta a mejorar la capacidad para saber, puesto que las conexiones facilitan el aprendizaje continuo, lo cual conlleva a ampliar la perspectiva entre campos, ideas y conceptos, los cuales deben, estar en permanente actualización. El aprendizaje trae consigo el manejo de toma de decisiones puesto que incita al aprendiz a determinar qué es lo que desea aprender (Martínez y Martínez, 2017). 


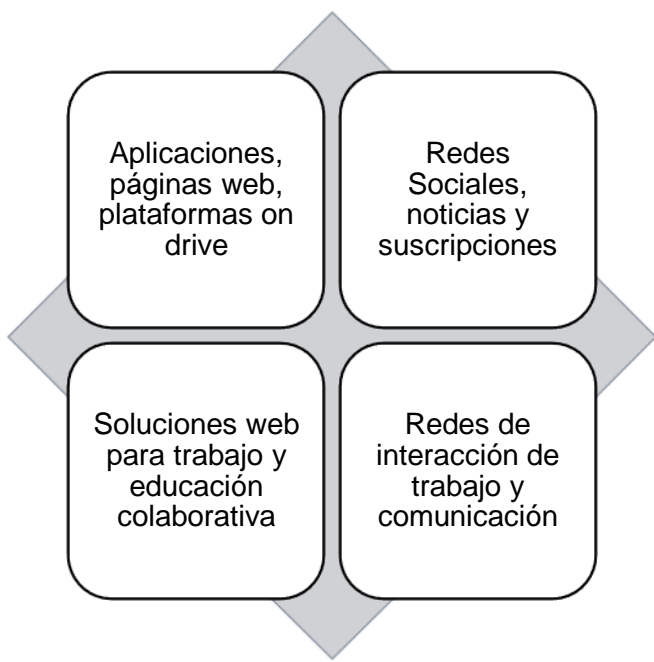

Fig. 2: Herramientas TIC, utilizadas en la educación virtual

\section{Principios del Conectivismo}

Según reflexiones del autor de esta teoría, este constructo tiene una serie de principios que conforma sus argumentos y son (Renó, 2012): 1) el aprendizaje y el conocimiento, necesitan de distintos juicios para ser; 2) el aprendizaje es el proceso de conectar fuentes de información; 3) el aprendizaje no es exclusivo de los seres humanos; 4) la disposición y la competencia para ampliar los saberes, se valoran más que el mismo conocimiento; 5) es indispensable proveer y conservar los vínculos entre áreas, ideas y conceptos; y 6) dentro de la ideología conectivista, la actualización de la información y conocimientos es fundamental en sus actividades.

\section{METODOLOGÍA}

Para alcanzar al objetivo planteado en la investigación se optó por una metodología cualitativa y cuantitativa que buscó determinar los niveles de posicionamiento conceptual de las estrategias pedagógicas por parte de los docentes y las percepciones que se tienen de las mismas. Este ejercicio, permitió realizar análisis en el software estadístico SPSS y generar ilustraciones que posibilitaron las reflexiones que la información aportada por los participantes pueda derivar en la generación de alternativas a tener en cuenta en futuras profundizaciones e investigaciones relacionadas. En total, fueron consultados 55 docentes de diversos programas en cuatro universidades de la ciudad de Barranquilla, que de forma voluntaria y amparados en la Ley 1266 de 2008 aportaron información vital para este proceso de análisis.

\section{RESULTADOS}

Se aprecia que los niveles de conocimiento se ubican en un $52.7 \%$ la categoría alta, el $45.5 \%$ en la categoría moderada y en bajo conocimiento el $1.8 \%$. Estos resultados permiten afirmar que, los conceptos asociados a estrategias pedagógicas se encuentran bien posicionados y que el nivel de desconocimiento no es relevante en el grupo de consultados. Estos resultados contrastan con lo expuesto por Allen y Seaman (2015) que indican que solo un $28 \%$ de los directivos universitarios consideran que los docentes aceptan y validan la legitimidad de la educación en línea, sin embargo, manifiestan que uno de sus objetivos principales, en el área de educación virtual, es experimentar con pedagogía innovadora. Por otro lado, Muñoz et al. (2015) manifiesta que las estrategias deben estar en sintonía con la gestión del conocimiento que genera en estos entornos, donde, desde un punto de vista ontológico, es necesario integrar estos procesos con la arquitectura tecnológica para su mejorar su eficacia.

Una vez definido el nivel de conocimiento de las estrategias pedagógicas, se indagó sobre tres componentes esenciales que las mismas traen incorporados y son: el social, cultural y tecnológico. Los tres elementos citados, fueron examinados, pues en la medida que los mismos logran una buena interacción deriva en resultados más destacables dentro del proceso y potencian los resultados en los procesos de educación superior de mediación virtual. Adicional a estos elementos, Miller (2015) indica que la persistencia es un factor significativo para alcanzar los objetivos de una pedagogía virtual, pues interviene la garantía de calidad y facilidad de uso técnico para que el estudiante se mantenga motivado.

Más allá del alto nivel de conocimiento evidenciado en los resultados, según Hood y Littlejohn (2017) para que el docente desarrolle y explote la gran cantidad de recursos que existen actualmente aplicables a las 
estrategias pedagógicas, es necesario que participen de manera activa en las actividades de aprendizaje, con lo cual se lograrían adaptar y personalizar las prácticas, de este modo, se va generando conocimiento a través de la praxis que se reutiliza y nuevamente en el proceso.

Por otra parte, Bruce (2016), expone que las estrategias de aprendizaje, en un contexto globalizado como el actual, no pueden estar divorciadas de los elementos de interculturalidad y sin una comprensión integral de lo internacional, dado que la diversidad culturar está impactando las necesidades de aprendizaje de las personas. En tal sentido, el dominio conceptual de las estrategias pedagógicas contiene elementos del entorno que forman parte integral de éstas. Seguidamente, los datos que se procesaron sobre los niveles de conocimientos en estrategias pedagógicas, se ilustran de acuerdo al contenido de la tabla 2, que se encuentra a continuación:

Tabla 2: Dominio Conceptual de Estrategias Pedagógicas

\begin{tabular}{|c|l|c|c|c|c|}
\hline \multicolumn{2}{|c|}{} & Frecuencia & Porcentaje & Porcentaje válido & Porcentaje acumulado \\
\hline \multirow{4}{*}{ Válido } & Conocimiento Moderado & 25 & 45,5 & 45,5 & 45,5 \\
\cline { 2 - 6 } & Alto Conocimiento & 29 & 52,7 & 52,7 & 98,2 \\
\cline { 2 - 6 } & Bajo Conocimiento & 1 & 1,8 & 1,8 & 100 \\
\cline { 2 - 6 } & Total & 55 & 100 & 100 & \\
\hline
\end{tabular}

De forma adicional y según los resultados compilados se puede deducir que el Componente 1 o denominado elemento social, tiene una alta participación o acogida, el Componente 2 o cultural plantea un nivel de aceptación moderado y finalmente el Componente 3 o tecnológico alcanza un nivel igualmente moderado. De esta información se desprenden orientaciones que sugieren, que los aspectos culturales asociados a los escenarios y estrategias de educación superior están a nivel medio y que en la medida que los componentes puedan ser proyectados igualmente serán estimuladas las estrategias pedagógicas.

\section{CONCLUSIONES}

En la actualidad la información y el conocimiento, representadas en los conceptos de sociedad de la información y sociedad del conocimiento, representan los nuevos factores de importancia para el desarrollo de la sociedad, complementariamente a los tradicionales tierra, capital y trabajo. Bajo este enfoque la educación y los programas de formación adquieren especial importancia puesto que deben orientarse a los cambios que estos nuevos factores generan en el desarrollo económico y social (Rozo, 2011).

Desde el paradigma constructivista el conocimiento se construye a través de procesos progresivos de desarrollo de ideas que impactan y son de importancia para una comunidad. Bajo esta perspectiva, alumnos y docentes deben involucrar con el fin de producir y mejorar el conocimiento entendiendo que los esfuerzos grupales generan mayor resultado que los esfuerzos individuales, por lo tanto, es un esfuerzo meramente colaborativo.

Existe un esfuerzo investigativo en la actualidad por comprender la forma en la que ocurre este proceso de construcción de conocimiento entre alumnos cuando realizan trabajos colaborativos (García y Pineda, 2010). Estos procesos educativos, específicamente la generación de conocimiento se produce entonces en un entorno globalizado, y que está totalmente interrelacionado.

Desde este enfoque, la globalización puede concebirse como un fenómeno que permite estar interconectados e informados y por lo tanto acceder a conocimiento desde cualquier lugar o plataforma disponible (Gómez, 2010). Con base en lo expuesto anteriormente y los hallazgos del estudio se desprenden algunas conclusiones importantes: 1). Existe un alto dominio conceptual de las estrategias pedagógicas en el grupo de docentes entrevistados, específicamente un 98,2\%, correspondiente a las categorías alto y moderado conocimiento. Esto permite contar con apertura y disposición del docente para innovar en las prácticas educativas. 2). Los resultados están en sintonía con las tendencias mundiales sobre la incorporación de estrategias pedagógicas que toman en cuenta la interculturalidad, factores sociales y la arquitectura tecnológica adecuada. 3). La calidad de las estrategias pedagógicas con mediación virtual se mide en función de las características técnicas del entorno, en términos de facilidad de uso y acceso, y el rol del docente como mediador y líder del proceso que estimule la participación y la comunicación fluida en el grupo. 4). La participación activa del docente en las actividades de aprendizaje permite la adopción, mejora y personalización de las estrategias pedagógicas. 


\section{REFERENCIAS}

Al-Atabi, M. y J. De Bóer, Teaching entrepreneurship using massive open online course (MOOC), Technovation, 34(4), 261-264 (2014)

Allen, I.E. y J. Seaman, Grade Level: Tracking Online Education in the United States, Babson Survey Research Group (2015) Aparici, R. y M. Silva, Pedagogía De La Interactividad, Comunicar, 19(38), 51-58 (2012)

Arroyo, L., P. Huertas, C. Peirano y M. Pérez, Las Habilidades del Pensamiento y el Aprendizaje Significativo en Matemática de Escolares de Quinto Grado en Costa Rica, Revista Electrónica " Actualidades Investigativas en Educación", 14(2), (2014)

Bartolomé, A. y K. Steffens, ¿Son los MOOC una Alternativa de Aprendizaje?, Comunicar, 22(44), 91-99 (2015)

Bolívar, C.R. y A.A. Dávila, Propuesta de Buenas Prácticas de Educación Virtual en el Contexto Universitario, Revista de Educación a Distancia, 49(12), 1-21 (2016)

Bottero, R.R., Educación y Pedagogía en el Marco del Neoliberalismo y la Globalización, Perfiles Educativos, 35(142), 149-166 (2013)

Bruce, A., Global Citizenship and Leadership in Changed Learning Environments, Re-Imagining Learning Scenarios, Annual conference, Socio-Economic and Cultural Aspects in e-Learning (2016)

Cardama, S.M. y M.C. Sebastián, La Teoría de la Complejidad en Biblioteconomía y Documentación: análisis y presencia en la investigación, Ibersid: revista de sistemas de información y documentación, 11(2), 13-22 (2017)

Cárdenas, I.R., M.G. Zermeño y R.F.A. Tijerina, Tecnologías Educativas y Estrategias Didácticas: Criterios de Selección, Revista Educación y Tecnología, 3, 190-206 (2013)

Costa, L.S., V.F. Barros, M.C. López y L.P. Marques, La Formación Docente y la Educación de Jóvenes y Adultos: Análisis de la Práctica Pedagógica para la Enseñanza de Ciencias, Formación Universitaria, 8(1), 03-12 (2015)

De Pinho, A.L.S., F.M. De Sales, J.G. Santa Rosa y M.A.S. Ramos, Technical and Pedagogical Usability in a Virtual Learning Environment: A Case Study at the Federal Institute of Rio Grande do Norte-Brazil, In Information Systems and Technologies (CISTI), 2015 10th Iberian Conference on, 1-4, IEEE (2015)

Díaz, L. y S. Omara, Prácticas Innovadoras de Enseñanza con Mediación TIC que Generan Ambientes Creativos de Aprendizaje, Revista Virtual Universidad Católica del Norte, 43(1), 147-160 (2014)

Dusell, I., Is School Curriculum Still Relevant in Digital Culture? Debates and Challenges on Contemporary Cultural Authority, Education Policy Analysis Archives, 22, 24 (2014)

Edel-Navarro, R., Entornos Virtuales de Aprendizaje: la Contribución de" lo Virtual" en la Educación, Revista mexicana de investigación educativa, 15(44), 7-15 (2010)

Fainholc, B., H. Nervi, R. Romero y C. Halal, La Formación del Profesorado y el Uso Pedagógico de las TIC, Revista de Educación a Distancia, (38) 01-14 (2015)

Flores, J., Los Recursos de Aprendizaje en la Educación a Distancia. El Paradigma Digital en Nuestro Diario Vivir, $1^{\text {a }}$ Ed., Fondo Editorial, 12-18, Lima, Perú (2014)

Fowler, C., Virtual Reality and Learning: Where is the Pedagogy? British Journal of Educational Technology, 46(2), 412422 (2015)

García, B. y V.J. Pineda, La Construcción de Conocimiento en Foros Virtuales de Discusión entre Pares, Revista mexicana de investigación educativa, 15(44), 85-111 (2010)

García, I., Emergent Leadership: Is E-Leadership Importance In The Quality Of Virtual Education? RIED, Revista Iberoamericana de Educación a Distancia, 18(1), 25-44 (2015)

Gil, A. y J. Roca, Movilidad Virtual, Reto del Aprendizaje de la Educación Superior en la Europa 2020, Revista de Educación a Distancia, (26), 01-16 (2011)

Gómez, B.R., Tendencias Actuales en la Educación Superior: Rumbos del Mundo y Rumbos del País, Revista Educación y pedagogía, 18(46), 79-90 (2010)

Gómez, G.M., M.A. Rolando y otros tres autores, Tendencias Metodológicas Universitarias en los Siglos XX y XXI. Estudio Comparativo University Methodological Trends in the Twenty and Twenty-First Centuries. A Comparative Study, Revista Ciencia Unemi, 9(17), 125-135 (2016)

Gomez, M.V., Use and Mastery of Virtual Learning Environment in Brazilian Open University, International Journal of Instruction, 7(2), 119-134 (2014)

Gutiérrez, A.F., G.M.B. Blanco y S.F. Méndez, Redes Sociales y Educación: el Análisis Socio-político como Asignatura Pendiente, Revista Interuniversitaria de Formación del Profesorado, 29(1), 119-129 (2015)

Henao, D., La teoría de Redes: Aspectos Claves para el Análisis de la "Fuga de Cerebros", Trans-pasando Fronteras, (2), 97-109 (2012)

Hood, N. y A. Littlejohn, Knowledge Typologies for Professional Learning: Educators' (re) Generation of Knowledge when Learning open Educational Practice, Educational Technology Research and Development, 65(6), 1583-1604 (2017) 
Ichimura, Y. y K. Suzuki, Dimensions of MOOCs for Quality Design: Analysis and Synthesis of the Literature, International Journal, 11(1), 42-49 (2017)

Marín, F.V., A.D.J. Inciarte, H.G. Hernández y R.C. Pitre, Estrategias de las Instituciones de Educación Superior para la Integración de las Tecnología de la Información y la Comunicación y de la Innovación en los Procesos de Enseñanza. Un Estudio en el Distrito de Barranquilla, Colombia, Formación Universitaria, 10(6), 29-38 (2017)

Martín, G.M., R.M. Martínez y otros tres autores, Acercamiento a las Teorías del Aprendizaje en la Educación Superior, UNIANDES EPISTEME, 4(1), 48-60 (2015)

Martinez, F.S. y A.G. Martinez, Fundamentals of Networked Learning Based on connectivism and Activity Theory, Revista Cubana de Educación Superior, 3(3), 98-112 (2017)

Miller, S.L., Teaching an Online Pedagogy MOOC, Journal of Online Learning \& Teaching, 11(1), (2015)

Muñoz, A., V. López y otros cuatro autores, Knowledge Management for Virtual Education Through Ontologies." OTM Confederated International Conferences" On the Move to Meaningful Internet Systems, Springer, Cham (2015)

Olea, F.P., C. Villacís y P.Á. Muñoz, ICT'S as a Tool in the Teaching-learning Process to Improve Academic Achievement, Revista Ciências Pedagógicas e Innovación, 3(1), 56-62 (2016)

Recino, U., O. Elizabeth y D. Ortega, Using the Flipped Classroom to Teach Educational Models in English at the Education National University (UNAE) of Ecuador, Speech, Language and Hearing, 1-4 (2017)

Renó, L., Transmedia, Conectivismo y Educación: Estudios de Caso. Campalans, C., D. Renó, Denis y V. Gosciola, Narrativa transmedia: entre teorías y prácticas, Bogotá: Editorial Universidad del Rosario (2012)

Rozo, A.C., Dimensión Pedagógica de la Educación Virtual: Una reflexión pendiente, Pedagogía y saberes, (32), 33-44 (2011)

Rubia, B., Revolution in Education? Computer Support for Collaborative Learning (CSCL), Comunicar, 21(42), 10-14 (2014)

Salguero, F.L. y J.R. Peña, The ICT Coordination and Teacher Education as Drivers of Educational Reform in the School, Enseñanza y Teaching, 33(2), 105 (2015)

Sierra, C.A., La Educación Virtual como Favorecedora del Aprendizaje Autónomo, Panorama, 5(9) 75-87 (2013)

Sobrino, A., Aportaciones del Conectivismo como Modelo Pedagógico Post-constructivista, Propuesta educativa, (42), 3948 (2014)

Toppin, I.N. y S.M. Toppin, Virtual Schools: The Changing Landscape of K-12 Education in the US, Education and Information Technologies, 21(6), 1571-1581 (2016)

Unzaga, M.F., J. Origlia y otros cuatro autores, La Universidad Argentina y el Impacto Económico del Conocimiento Globalizado; In IV Congreso Nacional y III Congreso Internacional de Enseñanza de las Ciencias Agropecuarias (2012)

Vázquez, E. y E. López, Los MOOC y la Educación Superior: la Expansión del Conocimiento, Revista Profesorado, 18(1), 01-12 (2014) 